\title{
Hepatic Nrf2 expression is altered by quercetin supplementation in $\mathrm{X}$-irradiated rats
}

\author{
RAQUEL MARINA ${ }^{1}$, PAQUITA GONZÁLEZ ${ }^{2}$, M. CARMEN FERRERAS ${ }^{3}$, \\ SERAFÍN COSTILLA $^{4}$ and JUAN-PABLO BARRIO ${ }^{2}$ \\ ${ }^{1}$ Universidad Autónoma de Chile, Santiago de Chile 7500566, Chile; Departments of ${ }^{2}$ Biomedical Sciences and \\ ${ }^{3}$ Animal Health, University of León, León 24071; ${ }^{4}$ Department of Medicine, University of Oviedo, Oviedo 33006, Spain
}

Received November 26, 2013; Accepted July 4, 2014

DOI: $10.3892 / \mathrm{mmr} .2014 .2741$

\begin{abstract}
Whole-body irradiation has been associated with liver function alterations. Ionizing radiation exposure increases oxidative stress and antioxidants can activate transcription of antioxidant target genes. In the present study, modifications of the liver antioxidant system were evaluated at 7 and 30 days following sub-lethal whole-body X-irradiation in male Wistar rats, which were intragastrically supplemented with quercetin or control solvent for 4 days prior to and 6 days following irradiation. Animal groups were as follows: CS, control, solvent-supplemented; CQ, control, quercetin-supplemented; RS, irradiated, solvent-supplemented; and RQ, irradiated, quercetin-supplemented. After 7 days, liver tissue from RS animals demonstrated marked hydropic panlobular degeneration with Mallory bodies in ballooning hepatocytes. These changes were mostly reversed in RQ rats. Lipid peroxidation in addition to copper/zinc superoxide dismutase (Cu/Zn-SOD), nuclear factor (erythroid-derived 2)-like 2 (Nrf2) and Kelch-like ECH-associated protein 1 (Keap1) protein expression levels were all increased by X-irradiation, but significantly decreased by quercetin supplementation. Catalase (CAT) and
\end{abstract}

Correspondence to: Dr Juan-Pablo Barrio, Department of Biomedical Sciences, University of León, Campus Universitario, León 24071, Spain

E-mail:.jpbarl@unileon.es

Abbreviations: ARE, antioxidant response element; CAT, catalase; $\mathrm{Cu} / \mathrm{ZnSOD}, \quad$ copper/zinc superoxide dismutase; EpRE, electrophile response element; Keap1, Kelch-like ECH-associated protein 1; MnSOD, manganese superoxide dismutase; NQO1: NAD(P)H: quinone oxidoreductase 1; Nrf2, nuclear factor (erythroid-derived 2)-like 2; PEG-CAT, polyethylene glycol-conjugated catalase; PEG-SOD, polyethylene glycol-conjugated superoxide dismutase; QQ, quercetin ortho-quinone/quinone methide; TBARS, thiobarbituric acid reactive substance

Key words: antioxidants, liver, Nrf2, oxidative stress, quercetin, radiation, rat
NAD(P)H: quinone oxidoreductase 1 (NQO1) expression levels remained high in irradiated rats regardless of quercetin supplementation. After 30 days, the liver from RS animals had small portal infiltrates and diffuse cytoplasmic vacuolization, with reduced lipid peroxidation and reduced expression levels of CAT, NQO1, Nrf2 and Keap1, but consistently elevated $\mathrm{Cu} / \mathrm{Zn}$-SOD expression. RQ animals indicated reduced expression levels of Nrf2 and Keap1 30 days after irradiation. The present study demonstrated a quercetin-induced reduction of the oxidative stress-associated increase in Nrf2 expression that may be useful for preventing cancer cell survival in response to ionizing radiation exposure.

\section{Introduction}

Polyphenols are antioxidant molecules that exist in a wide variety of fruits and vegetables. These compounds are able to interact with defense systems of the cell, and upregulate genes containing a cis-acting element in their promoter region known as antioxidant response element/electrophile response element (ARE/EpRE) (1). When oxidative stress occurs in the cells, nuclear factor (erythroid-derived 2)-like 2 (Nrf2) is released from the cytoplasm and translocates to the nucleus. Nrf2 binds the ARE in the regulatory regions of antioxidant target genes and activates transcription, which ultimately provides protection against a number of pathologies in various organs, including the liver, intestines, lungs, skin and nervous system (2).

Quercetin is one of the most frequently consumed dietary flavonoids, and serves multiple biologically significant functions, including antioxidant, anti-carcinogenic, anti-inflammatory, cardioprotective and radioprotective properties (3-5). Quercetin is able to affect the ARE activation pathway, and molecular studies have indicated the upregulation of Nrf2 through the regulation of both transcriptional and post-transcriptional sites of Nrf2. Enhanced repression of Kelch-like ECH-associated protein 1 (Keap1) was also demonstrated by affecting the post-transcriptional site, which revealed a number of substantial differences between oxidative inducers (1).

A plethora of studies associating antioxidant properties with the ability to reduce cytotoxic effects of ionizing radiation have been conducted. Healthy tissues can be damaged in 
multiple ways by radiation, depending on the type of cells and organs being irradiated, the dose and dose rate of radiation exposure, and the time elapsed from radiation exposure to the study of the effects. Chronic, low dose exposure to ionizing radiation can result in the induction of antioxidant enzymes, but not in a dose-dependent way. Otsuka et al (6) demonstrated that exposure of mice to $0.5 \mathrm{~Gy}$ at a dose rate of $1.2 \mathrm{mGy} / \mathrm{h}$ for 23 days increased the gene expression of catalase and MnSOD by a factor of 2.5 , while at higher doses of 1.0 and $1.3 \mathrm{~Gy}$ at a similar dose rate, gene expression either increased by only 1.4 or was not significantly different from non-irradiated controls, respectively.

The nucleus, cell membrane and mitochondria are considered sensitive targets for the reduction or prevention of radiation damage by antioxidants (7). One of the most studied effects of ionizing radiation is DNA damage. In order to exert preventive effects on the immediate genotoxicity induced by ionizing radiation, antioxidants must be able to access the nucleus and be near the DNA strands at the time of irradiation. Antioxidant agents may act via the scavenging of oxygen-based free radicals in addition to competing with oxygen for chemically repairing DNA damage by reacting with free radicals on the DNA (7). When ionizing radiation impacts on lipid membranes, the resulting increase in the formation of lipid radicals and peroxides can cause damage or the release of membrane proteins, or it can promote the liberation of lipid-based peroxidation products that will react with other cell structures (8). Long-term cell survival requires the availability of sufficient functional mitochondria to meet energy needs, and these organelles are particularly well-suited to withstand radiation damage due to their high antioxidant capacity and the fact that their DNA is located in multiple replicates (9).

Whole-body irradiation has been used in the clinical treatment of malignancies to induce immunosuppression and prevent allograft rejection, and it has been associated with significant changes in liver function (10). The aim of the present study was to evaluate the modifications in the liver antioxidant system by $\mathrm{X}$-irradiation in male Wistar rats under whole-body irradiation with a single sub-lethal dose, and the effects of quercetin supplementation prior to and following irradiation. The assessment was performed at 7 and 30 days following irradiation.

\section{Materials and methods}

Animals. Male Wistar rats (250 g), were initially housed in a room maintained at $22^{\circ} \mathrm{C}$ with a relative humidity ranging from 45 to $55 \%$ and a 12 -h dark/light cycle. The animals had free access to food (standard diet from Panlab, Barcelona, Spain) and water, and were not starved prior to experiments. All study protocols were reviewed and approved by the University of León Animal Care Committee (León, Spain) and were in accordance with the indications of the current Spanish and European laws (RD 53/2013 and EU Directive 2010/63/EU).

Experimental procedures. Animals were split into four groups of 8 , based on irradiation (non-irradiated controls and X-ray exposure group) and time periods of analysis ( 7 and 30 days). On the assumption that quercetin may have radiomitigative effects shortly following irradiation exposure (11), intragastric quercetin supplementation (50 mg/kg body weight) was used, following a previous study by Kawai et al (12). Thus, quercetin (50 $\mathrm{mg} / \mathrm{kg}$ body weight in propylene glycol) was administered intragastrically for 4 days prior to and 6 days following irradiation, and non-supplemented animals received the same volume of propylene glycol solvent alone. The groups were then constituted as the CS (controls, solvent-supplemented), CQ (controls, quercetin-supplemented), RS (irradiated, solvent-supplemented) and RQ (irradiated, quercetin-supplemented) groups.

Experimental irradiation with $X$-rays. RS and RQ animals were whole-body irradiated with a single X-ray dose of 6 Gy administered over $15 \mathrm{~min}$, at a source-skin distance of $50 \mathrm{~cm}$. In order to immobilize the animals, anesthesia was induced by intraperitoneal administration of pentobarbital $0.5 \%$ in saline (10 ml/kg body weight) at noon. This was $15 \mathrm{~min}$ prior to irradiation in order to ensure the loss of palpebral and plantar reflex activity, and spontaneous respiration throughout the procedure. The animals were positioned decubitus pronus on a plexiglas board, so that two animals were irradiated simultaneously. CS and CQ animals underwent the same procedure, excluding irradiation. The X-ray source was a Maxishot 200 (YXLON International GmbH, Hamburg, Germany) operated by qualified staff at the Instrumental Techniques Laboratory, University of León, in accordance with current Spanish legislation on radiation equipment use. X-ray filtration was accomplished in the Maxishot 200 machine according to manufacturer's instructions using 4-mm thick beryllium and $3-\mathrm{mm}$ thick aluminum filters in the X-ray tube unit. Uniform total-body X-irradiation distribution was confirmed by dosimetry using isodose curves for measurement. A test with a phantom (water layer) was performed in order to check self-shielding without changes in the dose distribution profile for the thickness involved.

Determination of lipid peroxidation. Aldehydic products generated by lipid peroxidation were determined by the thiobarbituric acid (TBA) reaction with malondialdehyde using the methods of a previous study (13). Liver (1 g) was homogenated in $9 \mathrm{ml}$ potassium phosphate $(0.1 \mathrm{M}, \mathrm{pH} 7.4)$ and tubes were prepared with $1 \mathrm{ml}$ fresh homogenate plus $2 \mathrm{ml}$ of a solution containing $15 \%$ trichloroacetic acid, $0.37 \%$ TBA, and $0.25 \mathrm{M} \mathrm{HCl}$. After $30 \mathrm{~min}$ at $90^{\circ} \mathrm{C}$, the tubes were cooled and centrifuged at 2,000 x g. Supernatants were collected and their absorbance read at $532 \mathrm{~nm}$.

Protein expression of copper/zinc superoxide dismutase $(C u / Z n-S O D)$, catalase $(C A T), N A D(P) H$ : quinone oxidoreductase 1 (NQO1), Nrf2 and Keapl. Liver tissue was cut into small pieces and homogenized in RIPA buffer [Tris- $\mathrm{HCl}$ $50 \mathrm{mM}$ pH 7.4, $\mathrm{KCl} 150 \mathrm{mM}$, sodium deoxycholate $0.5 \%$, NP-40 $0.1 \%$ and sodium dodecyl sulfate (SDS) 0.1\%] with protease/phosphatase cocktail (one tablet per $10 \mathrm{ml}$ RIPA buffer) (Roche Farma S.A., Madrid, Spain).

The cytoplasmic fraction was obtained as the supernatant from centrifugation of the liver extract $\left(1 \mathrm{~h}, 4^{\circ} \mathrm{C}, 12,000 \mathrm{x} \mathrm{g}\right)$. The nuclear fraction was obtained from fresh liver tissue, homogenized in buffer A (10 mM HEPES-NaOH pH 7.9, $1.5 \mathrm{mM} \mathrm{MgCl}{ }_{2}$ and $10 \mathrm{mM} \mathrm{KCl}$ ) including $0.1 \% \mathrm{NP}-40$ (EMD Millipore, Billerica, MA, USA) and centrifuged 
(10 min, $4^{\circ} \mathrm{C}, 1,000 \times \mathrm{g}$ ). The pellet was resuspended in buffer $\mathrm{A}$ without NP-40, centrifuged again, and then resuspended again in buffer B (20 mM HEPES-NaOH pH 7.9, glycerol, $420 \mathrm{mM}$ $\mathrm{NaCl}, 1.5 \mathrm{mM} \mathrm{MgCl}_{2}$ and $0.2 \mathrm{mM}$ EDTA $\mathrm{pH} 8.0$ ), prior to being centrifuged for $30 \mathrm{~min}$ at $4^{\circ} \mathrm{C}$ and $12,000 \mathrm{x} \mathrm{g}$.

Samples containing 50-100 $\mu \mathrm{g}$ protein were separated by SDS-polyacrylamide gel electrophoresis (12-14\% acrylamide) and transferred onto polyvinylidine fluoride membranes (EMD Millipore). The membranes were subsequently immersed in Ponceau stain in order to verify equal sample loading. Non-specific binding was blocked by pre-incubation of the membranes in phosphate-buffered saline containing 3-5\% non-fat dried milk for $30 \mathrm{~min}$ at $37^{\circ} \mathrm{C}$. Membranes were then incubated overnight at $4^{\circ} \mathrm{C}$ with polyclonal anti-Cu/Zn-SOD, anti-CAT, anti-NQO1, anti-Nrf2 and anti-Keap1 (Santa Cruz Biotechnology, Santa Cruz, CA, USA). Bound primary antibody was detected using a horseradish peroxidase-conjugated goat anti-mouse antibody (Dako, Glostrup, Denmark) by chemiluminescence using an Amersham ECL kit (GE Healthcare, Chalfont, UK) according to the manufacturer's instructions.

Histology. For histopathological examination, samples of the liver were trimmed and fixed by immersion in $10 \%$ buffered formalin for $24 \mathrm{~h}$. The blocks were dehydrated in a graded series of ethanol solutions (50, 70,96 and 100\%) and embedded in paraffin wax. Serial $4-\mu \mathrm{m}$ sections were stained with hematoxylin and eosin and evaluated blindly by a pathologist. Photomicrographs were performed on an Olympus Provis AX-70 microscope (Olympus Corporation, Tokyo, Japan) fitted with a DXM 1200 digital camera (Nikon Corporation, Tokyo, Japan).

Statistical analysis. Results are expressed as the mean \pm standard error. The data were compared by analysis of variance; significant differences in the means were compared with the Newman-Keuls test. Values were analyzed using the statistical package Statistica, version 8.0 (Statsoft, Inc., Tulsa, OK, USA).

\section{Results}

Histological findings. The livers of the CS group exhibited normal cell structure. In the RS group, the hepatocytes were swollen and enlarged (ballooned cells) 7 days following irradiation, due to marked hydropic degeneration, mainly in the mid-zonal and periportal areas. Hyaline inclusions (Mallory bodies) were observed within the ballooning hepatocytes. Occasional inflammatory cell infiltration (mainly lymphocytes) was observed in the portal triads of the RS group. The RQ group exhibited a reduction in hepatic pathological changes compared with the RS group, and mild hydropic change was observed in periportal hepatocytes. No significant hepatic pathological changes were observed in the CQ group compared with solvent-supplemented controls.

The hepatocellular degeneration in irradiated animals was more diffuse at 30 days post-irradiation, and some small portal infiltrates consisting mainly of lymphocytes were identified. Hepatocyte cytoplasm appeared diffusely vacuolized with a central nucleus, which is a feature compatible with fluid metabolism alteration (Fig. 1). Mitotic activity of hepatocytes and a high proportion of binucleate cells were evident. No differences were identified between RQ animals and the RS group. Thirty days following irradiation, untreated animals also displayed discrete portal infiltration with lymphocytes and macrophages. Hepatocytes, mainly from periportal areas, exhibited dense cytoplasmic inclusions, which may have been protein-based (hyaline droplets or Mallory bodies).

Oxidative stress markers. The cytoplasmic concentration of thiobarbituric acid reactive substance (TBARS), a marker for lipid peroxidation, was significantly increased in the RS group compared with the CS group, at 7 and 30 days post-irradiation (39.89 and $4.21 \%$, respectively). In RQ animals, the cytoplasmic concentration of TBARS increased to a lesser degree than in RS rats (Fig. 2).

Expression of antioxidant enzymes (CAT, Cu/ZN-SOD, NQO1). The expression of antioxidant enzymes was quantified by the measurement of protein levels using western blotting. CAT expression at 7 days post-irradiation was increased in irradiated animals, supplemented or unsupplemented with quercetin (47 and 53\%, respectively). No changes were observed in CAT enzyme expression at 30 days post-irradiation between any of the experimental groups. An increased expression level of $\mathrm{Cu} / \mathrm{Zn}$-SOD was observed in all irradiated animals (RS and RQ) at 7 and 30 days post-irradiation. This increase was significantly reduced by quercetin supplementation, but $\mathrm{Cu} / \mathrm{Zn}$-SOD expression did not return to control levels (Fig. 3). The expression of the NQO1 enzyme increased in all irradiated animals at 7 and 30 days post-irradiation with or without quercetin supplementation (Fig. 3).

Expression of transcription factor Nrf2 and protein Keapl. As presented in Fig. 4, irradiation in quercetin-supplemented and unsupplemented animals induced a significant increase (59 and $70 \%$, respectively) of nuclear expression levels of Nrf2 at 7 days post-irradiation. This activation was less marked at 30 days post-irradiation (Fig. 4, upper panel). By contrast, cytoplasmic Nrf2 expression levels remained unchanged throughout the experiment, irrespective of quercetin supplementation (Fig. 4, middle panel). The expression of Keap1 protein in the liver cytoplasmic fraction significantly increased in all irradiated animals (RQ and RS, vs. CS) at 7 and 30 days post-irradiation (Fig. 4). This increase in Keap1 protein expression level was reduced in RQ animals at 30 days, to a level that was not significantly higher than the level of the CS group.

\section{Discussion}

Experimental quercetin supplementation has been used to counteract oxidative stress in a number of forms, including dissolved in water (14), ethanol (15) or propylenglycol (12), or food-supplemented (12). Quercetin has been used as pre-treatment to radiation in mice (16) and other flavonoids have been tested both prior to and following irradiation (17). The present study has demonstrated the radiomitigative effects of quercetin when administered for four days prior to and six days following whole-body $\mathrm{X}$-irradiation. 

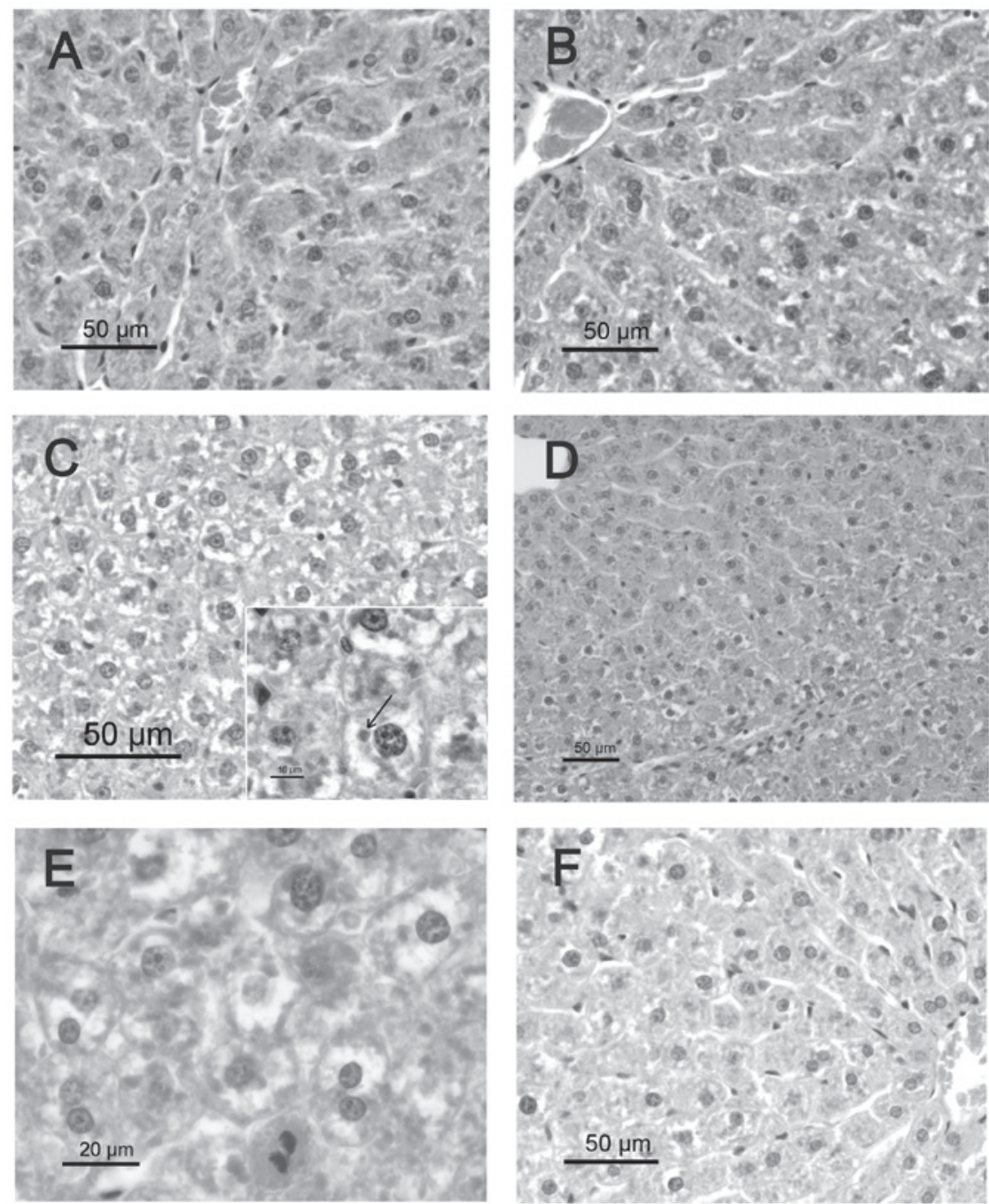

Figure 1. Liver parenchyma of (A) CS, (B) CQ, (C) RS (exhibiting diffuse cytoplasmic vacuolation. Arrow indicates the location of a Mallory body, magnification $\mathrm{x} 200$ ) and (D) RQ rat at 7 days post-irradiation, and a (E) RS and (F) RQ rat at 30 days post-irradiation. CS, control solvent-supplemented; CQ, control quercetin-supplemented; RS, irradiated solvent-supplemented; RQ, irradiated quercetin-supplemented.

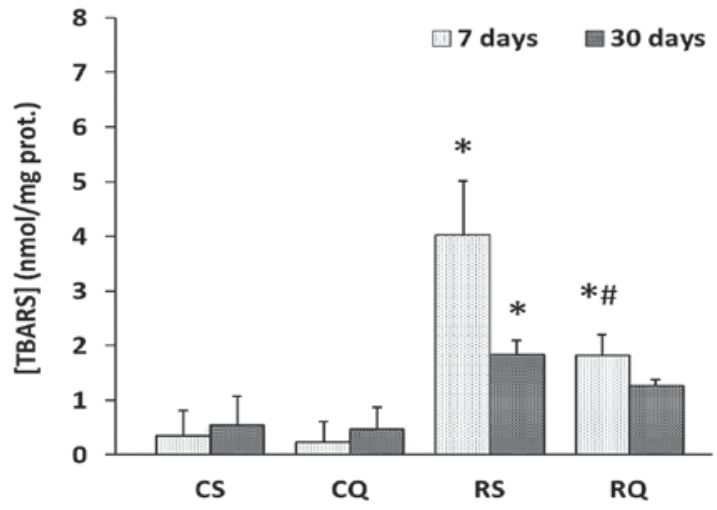

Figure 2. Lipid peroxidation in liver extracts obtained at 7 or 30 days post-irradiation. Data are presented as the mean value \pm standard error, $n=8$ in each group. ${ }^{*} \mathrm{P}<0.05$ vs. $\mathrm{CS},{ }^{*} \mathrm{P}<0.05$ vs. RS.

Hepatic injury induced by radiation is characterized by the loss of parenchymal hepatocytes and the distortion of the lobular architecture, which is accompanied by periportal fibrosis (18). Previous studies on human liver have indicated that the reaction of the liver to irradiation is largely dependent upon parameters such as the type of irradiation, dose, dose rate, fractionation schedule and irradiated volume, and the liver function is not normally compromised unless radiation doses $>35 \mathrm{~Gy}$ are used (19). The sensitivity of hepatocytes to ionizing radiation has been observed to be similar in human and rat liver tissues (20). The results of the present study suggest a marked hydropic degeneration at 7 or 30 days post-irradiation (6 Gy), which is most likely due to an intra-cytoplasmic accumulation of fluid as a result of the disturbed integrity of the cell membrane. Mallory bodies are accepted to represent degenerate cytoskeleton in damaged hepatocytes and are a consequence of cellular injury (21).

Quercetin supplementation may protect cells from the damage induced by ionizing radiation, since the data of the present study indicated that hydropic change was less extended and limited to periportal areas in quercetin-supplemented rats, compared with controls.

Ionizing radiation is established to induce oxidative stress through the generation of reactive oxygen species, resulting in an imbalance in the pro-oxidant/antioxidant status of the cells (22-24). Data of the current study indicate an apparent 

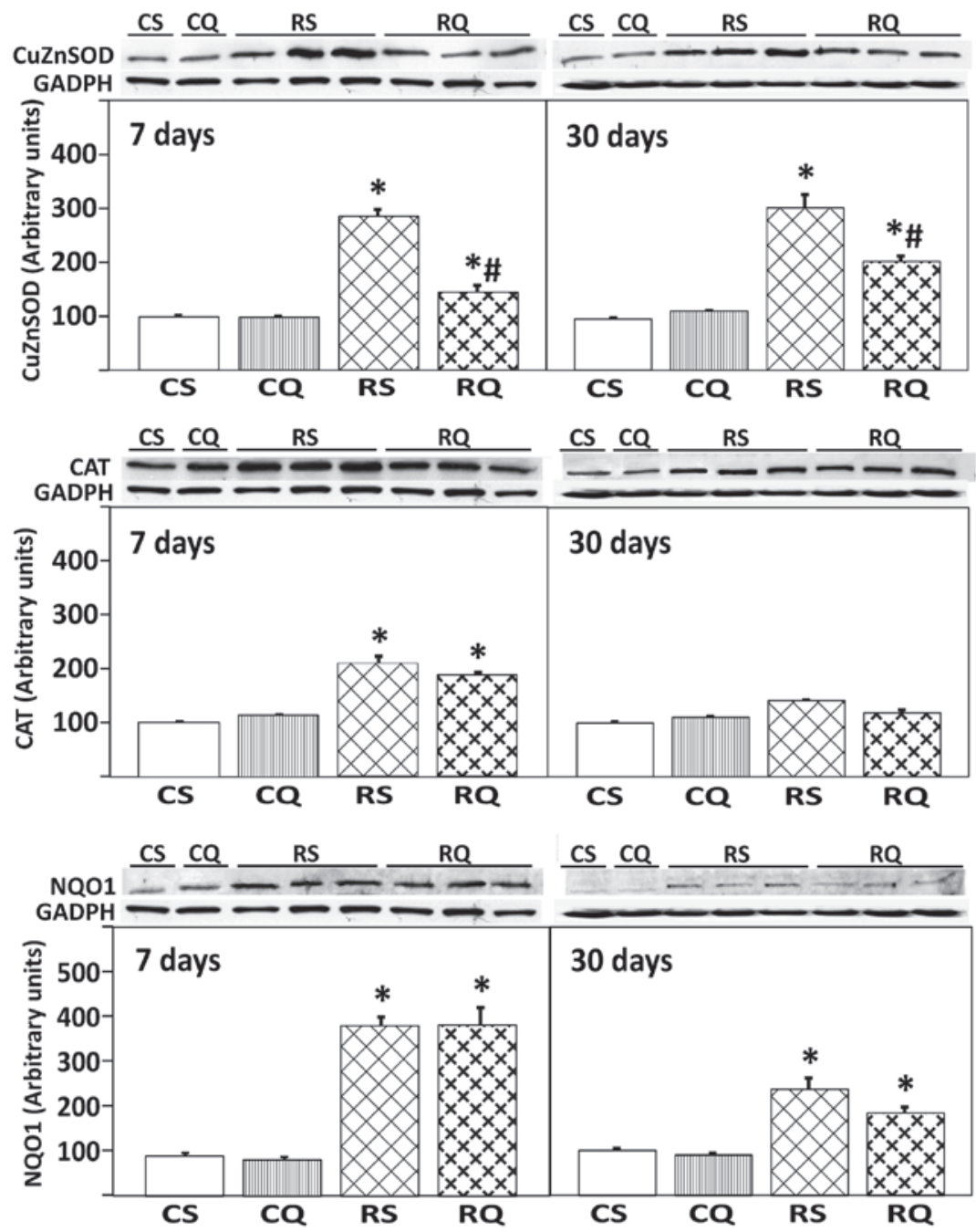

Figure 3. Protein expression of Cu/Zn-SOD, CAT and NQO1 in livers from the different experimental groups at 7 and 30 days following irradiation. At the top, representative Western blot photographs are shown. Data are presented as the mean value \pm standard error, $\mathrm{n}=8$ in each group. GADPH was used as loading control. ${ }^{*} \mathrm{P}<0.05$ vs. CS, ${ }^{\#} \mathrm{P}<0.05$ vs. RS. Cu/Zn-SOD, copper/zinc superoxide dismutase; CAT, catalase; NQO1, NAD(P)H: quinone oxidoreductase 1 ; CS, control solvent-supplemented; CQ, control quercetin-supplemented; RS, irradiated solvent-supplemented; RQ, irradiated quercetin-supplemented

increase in lipid peroxidation at 7 days post-irradiation. The presence of oxidative stress in the experimental model was confirmed by increased levels of TBARS in the liver in addition to the increase in the expression of the enzymatic antioxidant system. In the current results, lipid peroxidation reduced 30 days following irradiation to levels half of those observed 7 days following irradiation, but were still significantly higher than the non-irradiated controls. This is consistent with previous reports of decreased oxidative stress in rats 68 days after 8 Gy irradiation compared with 10 days post-irradiation (25). The present results indicate that quercetin may prevent oxidative stress, reducing the increase in lipid peroxidation markers and maintaining the expression of antioxidant enzymes.

Similar to previous reports (26), Nrf2 expression levels in the nuclear fraction were increased in response to $\mathrm{X}$-irradiation, confirming the important role of this nuclear factor in the antioxidant cell response. In contrast, no significant effects were observed following analysis of cytoplasmic Nrf2 expression levels. The present study also demonstrated that $\mathrm{X}$-irradiation is able to activate Nrf2 in order to increase
ARE-dependent gene expression 7 days following irradiation, but activated it to a lesser extent at 30 days post-irradiation. An accompanying increase in the expression of several antioxidant enzymes (CAT, Cu/Zn-SOD, NQO1) was also observed. Nrf2 expression levels were significantly reduced in the RQ animals compared with the RS group. These results may be interpreted as follows: By decreasing the levels of free radicals following quercetin supplementation, the activation of Nrf2 is reduced in accordance with the presently lower oxidation status. This idea is reinforced by the findings of McDonald et al (27) who indicated activation of Nrf2 following single doses of ionizing radiation from 2-8 Gy in breast cancer cells in a dose-dependent manner, but only following a delay of 5 days. As a result of the exogenous antioxidants glutathione and PEG-SOD (but not PEG-CAT) being administered post-irradiation, they demonstrated partially reduced ARE reporter activity after 5 days. This is also consistent with the finding of the present study, which indicated that $\mathrm{Cu} / \mathrm{Zn}$-SOD expression levels, but not those of CAT or NQO1, were reduced in RQ rats. However, other authors have reported increased Nrf2 expression levels following antioxidant administration. Liu et al (28) did not 

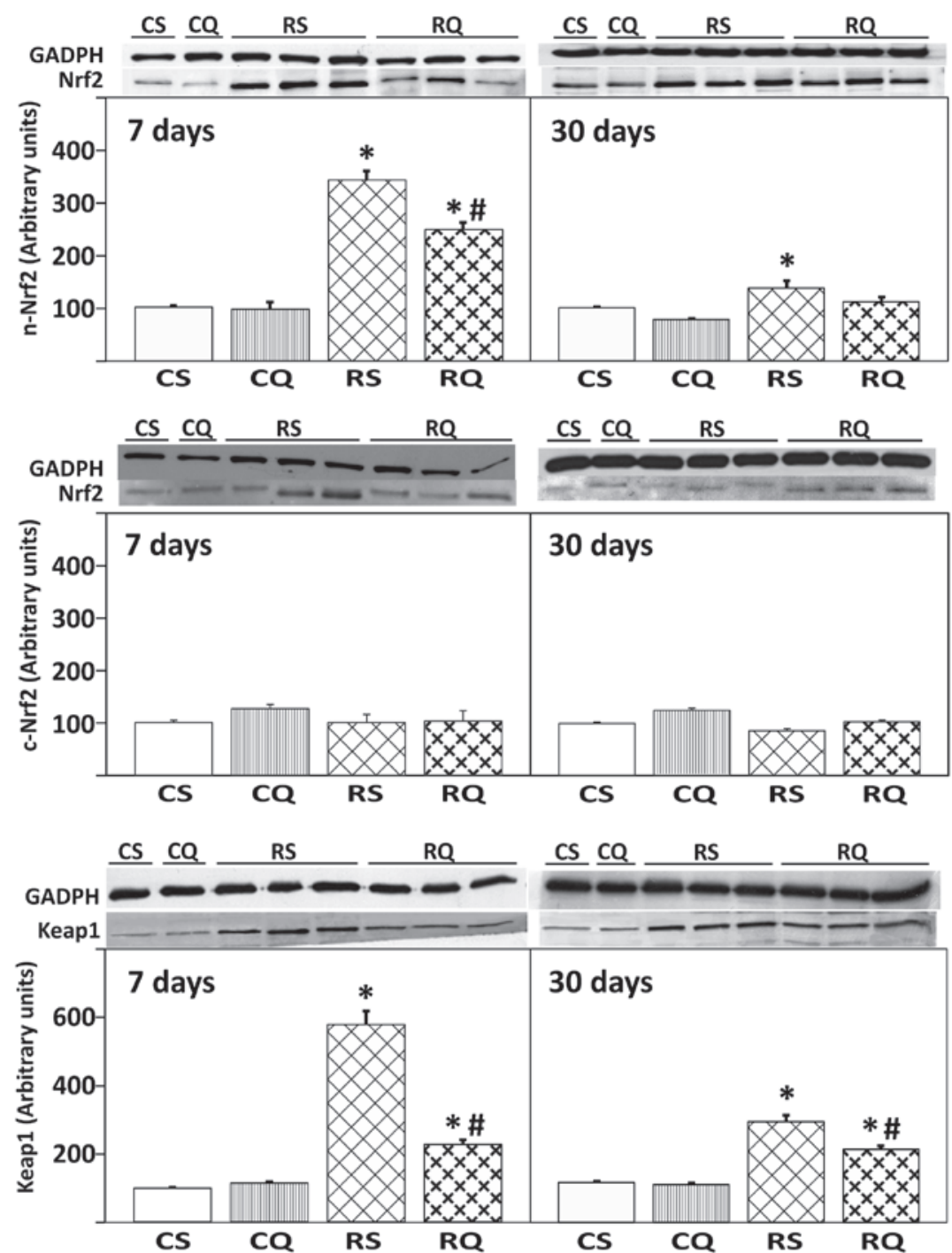

Figure 4. Protein expression of Nrf2 in cytoplasmic (c-Nrf2) and nuclear (n-Nrf2) compartments, and cytoplasmic Keap1 in livers from the four experimental groups at 7 and 30 days post-irradiation. At the top, representative western blot photographs are presented. Data are expressed as the mean value \pm standard error, $\mathrm{n}=8$ in each group. GADPH was used as loading control. ${ }^{*} \mathrm{P}<0.05$ vs. CS, ${ }^{*} \mathrm{P}<0.05$ vs. RS. Nrf2, nuclear factor (erythroid-derived 2)-like 2; Keap1, Kelch-like ECH-associated protein 1; CS, control solvent-supplemented; CQ, control quercetin-supplemented; RS, irradiated solvent-supplemented; RQ, irradiated quercetin-supplemented.

observe increased Nrf2 levels in mouse brain after $12 \mathrm{~h}$ of a single 4 Gy radiation dose, but did observe an increase with increasing concentrations of melatonin $(1-10 \mathrm{mg} / \mathrm{kg})$. Similarly, following dimethylnitrosamine-induced ROS increase, melatonin $(50 \mathrm{mg} / \mathrm{kg})$ administration over 14 days was able to double the nuclear levels of Nrf2 (29).

It is accepted that Keap1 is a protein involved in detecting oxidative stress via oxidative modification of distinct cysteine residues, leading to conformational changes that allow for the release of Nrf2, but Nrf2 itself may behave as a redox sensor. As the concentration of oxidants becomes higher, more Nrf2 proteins translocate into the nucleus at a higher speed. This graded nuclear translocation of Nrf2 indicates that Nrf2 can transmit the presence of oxidative stress and also the intensity of oxidative stress (30). An autoregulation interplay cycle has been reported between Nrf2 and Keap1, in that the increase in Nrf2 expression levels may induce the inducing activity of the Keap1 gene by binding to an ARE in the reverse strand of the proximal promoter of the Keap1 gene (31). This positive feedback may aid in explaining the parallel increases in nuclear Nrf2 and cytoplasmic Keap1 protein expression observed at 7 days post-irradiation, in addition to the reduced levels observed as a result of quercetin supplementation.

The $\mathrm{Cu} / \mathrm{Zn}$-SOD gene promoter contains an antioxidant responsive element (32). The transcription of the $\mathrm{Cu} / \mathrm{Zn}$-SOD gene has been reported to be upregulated in human HepG2 hepatoma cells following dioxin treatment used to induce ROS production and the activation of Nrf2 signalling (33), and also after irradiation in human oral cancer following preoperative radiation therapy (34) and human prostate cancer cells (35). In the results of the current study, the observed increase in $\mathrm{Cu} / \mathrm{Zn}$-SOD expression levels following whole-body irradiation is consistent with the activation of $\mathrm{Nrf} 2$ by increased oxidative stress. Quercetin supplementation reduced $\mathrm{Cu} / \mathrm{Zn}$-SOD expression levels, but they remained at a significantly higher level than non-irradiated controls, possibly as a consequence of the reduction in Nrf2 expression levels. 
The current study indicated that NQO1 expression was significantly increased at 7 days post-irradiation, and it remained unchanged following quercetin supplementation, while Nrf2 expression levels were significantly reduced in the RQ group. In order to explain these findings it may be important to consider the free radical-scavenging activity of quercetin, and that it produces oxidized derivatives such as quercetin ortho-quinone/quinone methide, which is a substrate that interacts with NQO1 (36). The fact that NQO1 expression was not altered in RQ rats may be attributed to the additional need for NQO1 to inactivate the potentially harmful oxidation products derived from quercetin antioxidant activity. Further research is required to clarify the dynamics of quercetin-derived adducts and their role in the antioxidant regulatory pathways.

The distinct changes in antioxidant enzyme expression observed in the present study can be interpreted by considering that ionizing radiation induces multiple molecular changes in the cells, depending on the dose and the time of testing. Transcription factors such as AP-1 and nuclear factor (NF)- $\mathrm{B}$ are activated only $24 \mathrm{~h}$ after irradiation (37), while MnSOD is simultaneously induced through the $N F-\kappa B$ pathway. It has also been reported that human lymphoblasts exposed at 3 Gy display increased levels of CAT and glutathione-peroxidase, but no other enzymes, at $20 \mathrm{~h}$ post-irradiation, suggesting that a low dose of radiation induces an increase in the expression of antioxidant enzymes as a radiomodifying response (38). Similarly, McDonald et al (27) demonstrated increased activation of Nrf2 after 5 days exposure of several cell lines to 8 Gy irradiation.

Quercetin has been successfully tested as an antioxidant in several in vitro studies using cell cultures $(16,39,40)$, but its in vivo activity has been questioned. Studying vitamin E-deficient rats supplemented with vitamin E or a variety of flavonoids, including quercetin, Duthie et al (41) concluded that in vivo supplementation with these antioxidant compounds (100 mg/kg diet) is ineffective in restoring lipid peroxidation indices in plasma and liver. However, another study has indicated that serum malondialdehyde levels are inversely correlated to the oral daily quercetin dose (0.01-1.00 g/kg body weight) after 22 days of treatment in otherwise normal Wistar rats (42); and this is in line with the results from the current study on lipid peroxidation.

Overexpression of $\mathrm{Nrf} 2$ can result in enhanced resistance of tumoral cells to chemotherapeutic agents, while downregulation of $\mathrm{Nrf} 2$ has been translated into more susceptibility to these drugs (43). These findings suggest that cancer cells can protect themselves from the stress-inducing conditions of the tumoral environment: An active Nrf2 pathway may promote a favorable redox balance and upregulate antioxidant gene products in order to enhance their survival; by contrast, normal cells do not have such high metabolic requirements and Nrf2 levels are not expected to be elevated to such a level. Supporting this view, in HepG2 cells incubated with 0-40 $\mu \mathrm{M}$ quercetin, this flavonoid enhanced the steady-state level of Nrf2 and also reduced the steady-state level of Keap1 through $26 \mathrm{~S}$ proteasome-independent degradation (1). The study also concluded that quercetin is able to increase the protein level of Nrf2 and simultaneously inhibit its ubiquitination, while Keap1 expression is reduced. While these effects have been observed in cell cultures without additional treatment, it has been recently reported that the combination of quercetin plus X-irradiation can significantly increase the tumoral radiosensitivity in vitro and in vivo (44). The data from the current study have demonstrated that quercetin in vivo supplementation influences the antioxidant system by reducing lipid peroxidation and modifying the activation of Nrf2 and various antioxidant enzymes. By administering quercetin to $\mathrm{X}$-irradiated animals, its antioxidant status was improved; the present study demonstrated that the oxidative stress-associated increase in Nrf2 expression levels in X-irradiated rats was diminished following quercetin supplementation. This approach could be utilized to avoid favoring cancer cell survival (by hindering the increase in Nrf2 expression levels), so quercetin may produce effects that reduce the oxidative stress-associated increase in Nrf2 expression levels, which may be beneficial in the prevention of cancer growth.

\section{Acknowledgements}

The authors are grateful for the financial support provided by Fundación Mapfre, and to Dra. María Teresa Ribas Ariño for her helpful assistance in histopathological interpretation.

\section{References}

1. Tanigawa S, Fujii M and Hou D: Action of Nrf2 and Keap1 in ARE-mediated NQO1 expression by quercetin. Free Rad Biol Med 42: 1690-1703, 2007.

2. Aleksunes LM and Manautou JE: Emerging role of Nrf2 in protecting against hepatic and gastrointestinal disease. Toxicol Pathol 35: 459-473, 2007.

3. Middleton EJ, Kandaswami C and Theoharides T: The effects of plants flavonoids on mammalian cells: implications for inflammation, heart disease, and cancer. Pharmacol Rev 52: 673-751, 2000.

4. González-Gallego J, García-Mediavilla MV, Sánchez-Campos S and Tuñón MJ: Fruit polyphenols, immunity and inflammation. Br J Nutr 104: S15-S27, 2010.

5. Hosseinimehr SJ: Trends in the development of radioprotective agents. Drug Discov Today 12: 794-805, 2007.

6. Otsuka K, Koana T, Tauchi H and Sakai K: Activation of antioxidative enzymes induced by low-dose-rate whole-body gamma irradiation: adaptive response in terms of initial DNA damage. Radiat Res 166: 474-478, 2006.

7. Okunieff P, Swarts S, Keng P, et al: Antioxidants reduce consequences of radiation exposure. Adv Exp Med Biol 614: 165-178, 2008.

8. Marnett LJ: Oxy radicals, lipid peroxidation and DNA damage. Toxicology 181-182: 219-222, 2002.

9. Wallace DC: The mitochondrial genome in human adaptive radiation and disease: on the road to therapeutics and performance enhancement. Gene 354: 169-180, 2005.

10. Nwokocha CR, Nwokocha M, Mounmbegna P, et al: Proteins and liver function changes in rats following cumulative total body irradiations. West Indian Med J 61: 773-777, 2012.

11. Citrin D, Cotrim AP, Hyodo F, et al: Radioprotectors and mitigators of radiation-induced normal tissue injury. Oncologist 15: 360-371, 2010.

12. Kawai Y, Saito S, Nishikawa T, et al: Different profiles of quercetin metabolites in rat plasma: comparison of two administration methods. Biosci Biotechnol Biochem 73: 517-523, 2009.

13. Willis ED: Evaluation of lipid peroxidation in lipids and biological membranes. In: Biochemical Toxicology: A Practical Approach. Snell KB, Mullock B (eds.) IRL Press, Oxford, pp407-420, 1987.

14. Jung JH, Kang JI and Kim HS: Effect of quercetin on impaired immune function in mice exposed to irradiation. Nutr Res Pract 6: 301-307, 2012.

15. Kanter M, Aktas C and Erboga M: Protective effects of quercetin against apoptosis and oxidative stress in streptozotocin-induced diabetic rat testis. Food Chem Toxicol 50: 719-25, 2012. 
16. Benkovic V,Knezevic AH, Dikic D, et al: Radioprotective effects of quercetin and ethanolic extract of propolis in gamma-irradiated mice. Arh Hig Rada Toksikol 60: 129-138, 2009.

17. Sezer A, Usta U, Kozak Z and Yagci MA: The effect of a flavonoid fractions diosmin + hesperidin on radiation-induced acute proctitis in a rat model. J Cancer Res Ther 7: 152-156, 2011.

18. An JH, Kim J and Seong J: Redox signaling by ionizing radiation in mouse liver. Ann NY Acad Sci 1030: 86-94, 2004.

19. Cromheecke M, Konings AW, Szabo BG and Hoekstra HJ: Liver tissue tolerance for irradiation: experimental and clinical investigations. Hepatogastroenterology 47: 1732-4170, 2000.

20. Alati T, Van Cleef M, Strom SC and Jirtle RL: Radiation sensitivity of adult human parenchymal hepatocytes. Radiat Res 115 152-160, 1988

21. PeiRJ,Danbara N,Tsujita-KyutokuM,etal:Immunohistochemical profiles of Mallory body by a panel of anti-cytokeratin antibodies. Med Electron Microsc 37: 114-118, 2004.

22. Bhosle SM, Huilgol NG and Mishra KP: Enhancement of radiation-induced oxidative stress and cytotoxicity in tumor cells by ellagic acid. Clin Chim Acta 359: 89-100, 2005.

23. Karran P: DNA double strand break repair in mammalian cells. Curr Opin Genetics Dev 10: 144-150, 2000.

24. Andrade ER, Cruz IB, Andrade VV, et al: Evaluation of the potential protective effects of ad libitum black grape juice against liver oxidative damage in whole-body acute X-irradiated rats. Food Chem Toxicol 49: 1026-1032, 2011.

25. Meydan D, Gursel B, Bilgici B, Can B and Ozbek N: Protective effect of lycopene against radiation-induced hepatic toxicity in rats. J Int Med Res 39: 1239-1252, 2011.

26. Tsukimoto M, Tamaishi N, Homma T and Kojima S: Low-dose gamma-ray irradiation induced translocation of Nrf2 into nuclear in mouse macrophague RAW264.7. J Radiat Res 51: 349-353, 2010

27. McDonald JT, Kim K, Norris AJ, et al: Ionizing radiation activates the Nrf2 antioxidant response. Cancer Res 70: 8886-8895, 2010.

28. Liu Y, Zhang L, Zhang H, et al: Exogenous melatonin modulates apoptosis in the mouse brain induced by high-LET carbon ion irradiation. J Pineal Res 52: 47-56, 2012.

29. Jung KH, Hong SW, Zheng HM, Lee DH and Hong SS: Melatonin downregulates nuclear erythroid 2-related factor 2 and nuclear factor-kappaB during prevention of oxidative liver injury in a dimethylnitrosamine model. J Pineal Res 47: 173-183, 2009.

30. Li W, Khor TO, Xu C, et al: Activation of Nrf2-antioxidant signaling attenuates NFkappaB-inflammatory response and elicits apoptosis. Biochem Pharmacol 76: 1485-1489, 2008.

31. Lee OH, Jain AK, Papusha V and Jaiswal AK: An auto-regulatory loop between stress sensors INrf 2 and Nrf2 controls their cellular abundance. J Biol Chem 282: 36412-36420, 2007.
32. Milani P, Gagliardi S, Cova E and Cereda C: SOD1 Transcriptional and Posttranscriptional Regulation and Its Potential Implications in ALS. Neurol Res. Int 2011: 458427, 2011.

33. Park EY and Rho HM: The transcriptional activation of the human copper/zinc superoxide dismutase gene by 2,3,7,8-tetrachlorodibenzo-p-dioxin through two different regulator sites, the antioxidant responsive element and xenobiotic responsive element. Mol Cell Biochem 240: 47-55, 2002.

34. Terakado N, Shintani S, Nakahara Y, et al: Expression of $\mathrm{Cu} / \mathrm{Zn}-\mathrm{SOD}, \mathrm{Mn}-\mathrm{SOD}$ and GST-pi in oral cancer treated with preoperative radiation therapy. Oncol Rep 7: 1113-1117, 2000.

35. Vucic V, Isenovic ER, Adzic M, Ruzdijic S and Radojcic MB: Effects of gamma-radiation on cell growth, cycle arrest, death, and superoxide dismutase expression by DU 145 human prostate cancer cells. Braz J Med Biol Res 39: 227-236, 2006.

36. Boots AW, Bast A and Haenen GR: No role of DT-diaphorase (NQO1) in the protection against oxidized quercetin. FEBS Lett 579: 677-82, 2005.

37. Orlowski RZ and Baldwin AS Jr: NF-kappaB as a therapeutic target in cancer. Trends Mol Med 8: 385-389, 2002.

38. Bravard A,Luccioni C, Moustacchi E and Rigaud O: Contribution of antioxidant enzymes to the adaptative response to ionizing radiation of human lymphoblasts. Int J Radiat Biol 75: 639-645, 1999.

39. Crespo I, Garcia-Mediavilla MV, Almar M, et al: Differential effects of dietary flavonoids on reactive oxygen and nitrogen species generation and changes in antioxidant enzyme expression induced by proinflammatory cytokines in Chang Liver cells. Food Chem Toxicol 46: 1555-1569, 2008.

40. Kook D, Wolf AH, Yu AL, et al: The protective effect of quercetin against oxidative stress in the human RPE in vitro. Invest Ophthalmol Vis Sci 49: 1712-1720, 2008.

41. Duthie G and Morrice P: Antioxidant capacity of flavonoids in hepatic microsomes is not reflected by antioxidant effects in vivo. Oxid Med Cell Longev 2012: 165127, 2012.

42. Nakamura Y, Ishimitsu S and Tonogai Y: Effects of quercetin and rutin on serum and hepatic lipid concentrations, fecal steroid excretion and serum antioxidant properties. J Health Sci 46: 229-240, 2000

43. Wang XJ, Sun Z, Villeneuve NF, et al: Nrf2 enhances resistance of cancer cells to chemotherapeutic drugs, the dark side of Nrf2. Carcinogenesis 29: 1235-1243, 2008.

44. Lin C, Yu Y, Zhao HG, et al: Combination of quercetin with radiotherapy enhances tumor radiosensitivity in vitro and in vivo. Radiother Oncol 104: 395-400, 2012. 